\title{
Integration Processes Based on Radau Quadrature Formulas
}

\author{
By J. C. Butcher
}

1. Introduction. In a previous paper [1] certain properties of Runge-Kutta processes were derived. As an example of the use of these results a three stage implicit process was found in which the 17 conditions necessary for fifth-order accuracy were satisfied. Since this was achieved by a special choice of only 12 parameters the existence of various relationships between the equations that must be satisfied is suggested.

Subsequently some of these relationships were found [2] and a set of processes was derived such that the process of $\nu$ stages has order of accuracy $\rho=2 \nu$. The associated quadrature formula in this case is the $\nu$-point Gauss-Legendre formula. In this paper we shall refer to this type of integration process as a G-process.

In a $\mathrm{G}$-process the vectors $\mathbf{g}^{(1)}, \mathbf{g}^{(2)}, \cdots, \mathbf{g}^{(\nu)}$ which correspond to the different stages of the process are defined only implicitly and in practice they would need to be evaluated iteratively. The subject of the present paper, another class of implicit Runge-Kutta process, is based on the Radau quadrature formulas [3]. It will be seen that these have certain advantages over the G-processes in that the accuracy achieved for the same number of implicit stages is higher.

Results proved in [1] and [2] will be assumed here and the same notations will be used.

2. Processes based on Radau formulas. If $c_{1}=a_{11}=a_{12}=\cdots=a_{1 \nu}=0$ then the equation

$$
\sum_{j=1}^{\nu} a_{1 j} c_{j}^{k-1}=c_{1}^{k} / k
$$

is satisfied for any $k$. Hence if the first stage of a process is explicit this is consistent with the statement symbolized (in the notation of [2]) by $C(\xi)$ for any $\xi$. Similarly if $c_{\nu}=1, a_{1 \nu}=a_{2 \nu}=\cdots=a_{\nu \nu}=0$ the final stage is explicit and this is consistent with the statement $D(\xi)$ for any $\xi$.

In view of these considerations we are led to consider three types of quadrature formulas,

$\mathrm{I}: c_{1}=0, c_{2}, c_{3}, \cdots, c_{\nu}$ chosen so that $B(\xi)$ for $\xi$ as high as possible,

II : $c_{\nu}=1, c_{1}, c_{2}, \cdots, c_{\nu-1}$ chosen so that $B(\xi)$ for $\xi$ as high as possible,

III: $c_{1}=0, c_{\nu}=1, c_{2}, c_{3}, \cdots, c_{\nu-1}$ chosen so that $B(\xi)$ for $\xi$ as high as possible. It can be shown [3] that $c_{1}, c_{2}, \cdots, c_{\nu}$ are the roots of the equations

$$
\begin{aligned}
& \text { I: }\left(\frac{d}{d c}\right)^{\nu-1}\left\{c^{\nu}(1-c)^{\nu-1}\right\}=0, \\
& \text { II: }\left(\frac{d}{d c}\right)^{\nu-1}\left\{c^{\nu-1}(1-c)^{\nu}\right\}=0,
\end{aligned}
$$

Received 25 February 1963, revised May 9, 1963. 
III : $\left(\frac{d}{d c}\right)^{\nu-2}\left\{c^{\nu-1}(1-c)^{\nu-1}\right\}=0$,

respectively.

In each case $b_{1}, b_{2}, \cdots, b_{\nu}$ can be found from the linear equations $B(\nu)$ and this implies $B(2 \nu-1)$ for cases I and II or $B(2 \nu-2)$ for case III.

The existence and uniqueness of G-processes was shown in theorems 8, 9, 10 of [2]. Corresponding results will now be stated for integration process based on the three types of Radau formulas. Each theorem is stated in three parts and it is to be understood that the values of $c_{1}, c_{2}, \cdots, c_{\nu}, b_{1}, b_{2}, \cdots, b_{\nu}$ are chosen corresponding to the appropriate Radau formula. Also in parts I, III it is to be assumed that $a_{11}=a_{12}=\cdots=a_{1 \nu}=0$ and in parts II, III that $a_{1 \nu}=a_{2 \nu}=\cdots=a_{\nu \nu}=0$.

THEOREM 1.

$$
\begin{aligned}
& \text { I: If } A(2 \nu-1) \text { then } C(\nu), D(\nu-1), \\
& \text { II: If } A(2 \nu-1) \text { then } C(\nu-1), D(\nu) \text {, } \\
& \text { III: If } A(2 \nu-2) \text { then } C(\nu-1), D(\nu-1) \text {. } \\
& \text { TheOREM } 2 .
\end{aligned}
$$

I: Either of $C(\nu), D(\nu-1)$ implies the other and either implies $A(2 \nu-1)$,

II: Either of $C(\nu-1), D(\nu)$ implies the other and either implies $A(2 \nu-1)$,

III: Either of $C(\nu-1), D(\nu-1)$ implies the other and either implies $A(2 \nu-2)$.

THEOREM 3.

$\mathrm{I}: C(\nu)$ or $D(\nu-1)$ defines $a_{i j}(i=2, \cdots, \nu ; j=1, \cdots, \nu)$ uniquely,

II: $C(\nu-1)$ or $D(\nu)$ defines $a_{i j}(i=1, \cdots, \nu ; j=1, \cdots, \nu-1)$ uniquely,

III: $C(\nu-1)$ or $D(\nu-1)$ defines $a_{i j}(i=2, \cdots, \nu ; j=1, \cdots, \nu-1)$ uniquely.

The proofs of these results are almost identical with those of theorems 8,9 , 10 of [2]. However, when Theorem 5 of [2] would be used in parts II or III of a proof it should be replaced by

$$
\text { (1) If } B(\nu-1+\eta), E(\nu-1, \eta) \text { and } a_{1 \nu}=a_{2 \nu}=\cdots=a_{\nu \nu}=0 \text {, then } C(\eta) \text {. }
$$

Also when Theorem 6 of [2] would be used in parts I or III of a proof it should be replaced by

$$
\text { (2) If } B(\xi+\nu-1), E(\xi, \nu-1) \text { and } a_{11}=a_{12}=\cdots=a_{1 \nu}=0 \text {, then } D(\xi) \text {. }
$$

The statements (1) and (2) are proved in the same way as Theorems 5 and 6 of [2].

In the rest of this paper the three types of integration processes will be referred to as I-processes, II-processes, or III-processes.

For G-processes the values $c_{1}, c_{2}, \cdots, c_{\nu}$ (and hence $b_{1}, b_{2}, \cdots, b_{\nu} ; a_{11}, a_{12}$, $\cdots, a_{\nu \nu}$ ) could be expressed in terms of quadratic surds for $\nu=1,2, \cdots, 5$. For I-processes the same is true for $\nu=1,2,3$; for II-processes for $\nu=2,3$; and for III-processes for $\nu=2,3, \cdots, 7$. In these cases the values of the parameters are shown in Tables 1, 2, 3 corresponding to the three types of processes. For higher $\nu$ it seems to be more convenient to evaluate the parameters numerically. For $\nu=1$ there is neither a II-process nor a III-process. Numerical values of the parameters will form the subject of a later paper.

The I-process with $\nu=1$ is simply Euler's process (described in [4]); that with $\nu=2$ is the same as one due to Hammer and Hollingsworth [5]; that with 
TABle 1

\begin{tabular}{lcc|c|c}
$\nu=1:$ & $\frac{0}{1} \mid \underline{0}$ \\
$\nu=2:$ & 0 & 0 & \\
$\nu=3:$ & 0 & $\frac{\frac{1}{3}}{\frac{1}{3}}$ & $\frac{2}{3}$ & \\
& & 0 & & \\
& $\frac{9+\sqrt{6}}{75}$ & $\frac{24+\sqrt{6}}{120}$ & $\frac{168-73 \sqrt{6}}{600}$ & $\frac{6-\sqrt{6}}{10}$ \\
& $\frac{9-\sqrt{6}}{75}$ & $\frac{168+73 \sqrt{6}}{600}$ & $\frac{24-\sqrt{6}}{120}$ & $\frac{6+\sqrt{6}}{10}$ \\
\hline & $\frac{1}{9}$ & $\frac{16+\sqrt{6}}{36}$ & $\frac{16-\sqrt{6}}{36}$ & \\
\hline
\end{tabular}

TABLE 2

$$
\nu=2 \text { : }
$$$$
\begin{array}{ll|l}
\frac{1}{3} & 0 & \frac{1}{3} \\
1 & 0 & 1 \\
\hline \frac{3}{4} & \frac{1}{4} &
\end{array}
$$

$\nu=3:$

\begin{tabular}{ccc|c}
$\frac{24-\sqrt{6}}{120}$ & $\frac{24-11 \sqrt{6}}{120}$ & 0 & $\frac{4-\sqrt{6}}{10}$ \\
$\frac{24+11 \sqrt{6}}{120}$ & $\frac{24+\sqrt{6}}{120}$ & 0 & $\frac{4+\sqrt{6}}{10}$ \\
$\frac{6-\sqrt{6}}{12}$ & $\frac{6+\sqrt{6}}{12}$ & 0 & 1 \\
$\frac{16-\sqrt{6}}{36}$ & $\frac{16+\sqrt{6}}{36}$ & $\frac{1}{9}$ &
\end{tabular}

$\nu=3$ was previously published by the present author [1]. The III-process with $\nu=2$ is the improved Euler (or Heun) method [4]; the III-process with $\nu=3$ was quoted without detailed derivation by the present author [2].

3. The Error Term. In the same way as for G-processes, the principal error function $h^{\rho+1} \sum_{r=\rho+1} \epsilon \mathbf{F}$, where

$$
\delta=\Phi-\frac{1}{\gamma} \quad \epsilon=\frac{\beta \delta}{\rho !}
$$

can be found. For I-processes and II-processes, $\rho=2 \nu-1$ while for III-processes, $\rho=2 \nu-2$. The central elementary differentials of order $2 v-1$ will be found to play the same type of role here as with the G-process with $\nu-1$ stages. For the 


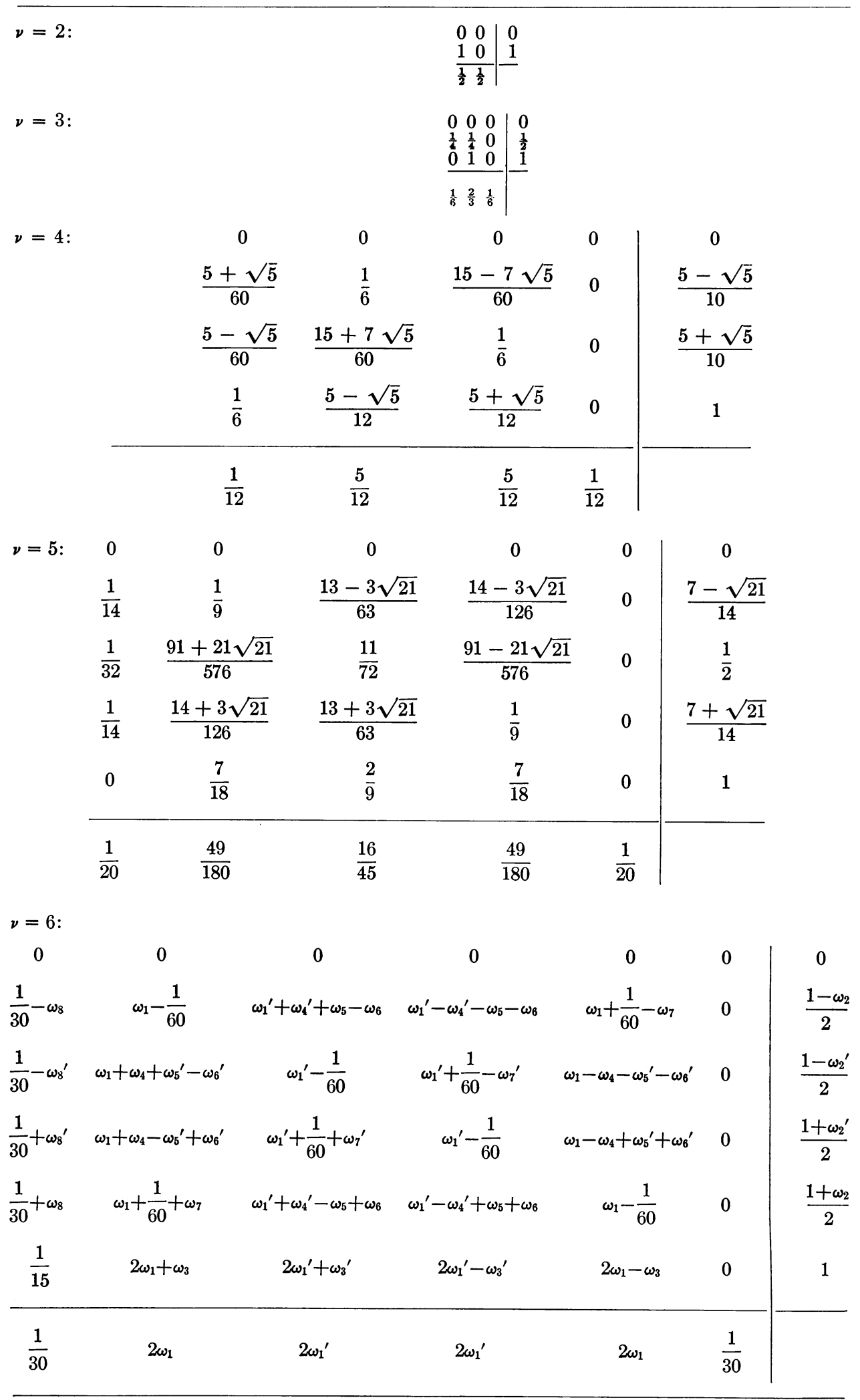


TABLE 3-Continued

$$
\begin{aligned}
& {\left[\omega_{1}=\frac{14-\sqrt{7}}{120}, \quad \omega_{1}^{\prime}=\frac{14+\sqrt{7}}{120}, \quad \omega_{2}=\sqrt{\frac{7+2 \sqrt{7}}{21}}, \quad \omega_{2}{ }^{\prime}=\sqrt{\frac{7-2 \sqrt{7}}{21}},\right.} \\
& \omega_{3}=\frac{7-5 \sqrt{7}}{60} \omega_{2}, \quad \omega_{3}{ }^{\prime}=\frac{7+5 \sqrt{7}}{60} \omega_{2}{ }^{\prime}, \quad \omega_{4}=\frac{2+5 \sqrt{7}}{120} \omega_{2}, \quad \omega_{4}{ }^{\prime}=\frac{2-5 \sqrt{7}}{120} \omega_{2}{ }^{\prime}, \\
& \omega_{5}=\frac{14+\sqrt{7}}{3780} \sqrt{21}, \quad \omega_{5}^{\prime}=\frac{14-\sqrt{7}}{3780} \sqrt{21}, \quad \omega_{6}=\frac{22+17 \sqrt{7}}{360} \omega_{2}, \quad \omega_{6}^{\prime}=\frac{22-17 \sqrt{7}}{360} \omega_{2}^{\prime}, \\
& \left.\omega_{7}=\frac{22-5 \sqrt{7}}{60} \omega_{2}, \quad \omega_{7}^{\prime}=\frac{22+5 \sqrt{7}}{60} \omega_{2}^{\prime}, \quad \omega_{8}=\frac{1-\sqrt{7}}{90} \omega_{2}, \quad \omega_{8}^{\prime}=\frac{1+\sqrt{7}}{90} \omega_{2}^{\prime}\right] .
\end{aligned}
$$$$
\nu=7:
$$

$$
0
$$

0

$\omega_{4} \quad \omega_{1}-\frac{1}{84}$

0

0

0

0

0

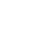$$
\omega_{4}^{\prime} \quad \omega_{3}{ }^{\prime}-\omega_{5}{ }^{\prime}+\omega_{6}
$$$$
\omega_{3}-\omega_{5}+\omega_{6}^{\prime}
$$$$
\omega_{7}-\omega_{8} \quad \omega_{3}-\omega_{5}-\omega_{6}^{\prime} \quad \omega_{1}-\frac{1}{84}-\omega_{9} \quad 0
$$$$
\omega_{1}^{\prime}-\frac{1}{84} \quad \omega_{7}^{\prime}-\omega_{8}^{\prime} \quad \omega_{1}^{\prime}-\frac{1}{84}-\omega_{9}^{\prime} \quad \omega_{3}^{\prime}-\omega_{5}^{\prime}-\omega_{6} \quad 0
$$$$
\frac{1}{32} \quad \omega_{10}+\omega_{11} \quad \omega_{10}^{\prime}+\omega_{11}^{\prime} \quad \frac{11}{100} \quad \omega_{10}^{\prime}-\omega_{11}^{\prime} \quad \omega_{10}-\omega_{11} \quad 0
$$$$
\omega_{4}^{\prime} \quad \omega_{3}^{\prime}+\omega_{5}^{\prime}+\omega_{6} \quad \omega_{1}^{\prime}-\frac{1}{84}+\omega_{9}^{\prime} \quad \omega_{7}^{\prime}+\omega_{8}^{\prime} \quad \omega_{1}^{\prime}-\frac{1}{84} \quad \omega_{3}^{\prime}+\omega_{5}^{\prime}-\omega_{6} \quad 0 \quad 0 \quad \frac{1+\omega_{2}^{\prime}}{2}
$$$$
\begin{array}{cccccccc}
\omega_{4} & \omega_{1}-\frac{1}{84}+\omega_{9} & \omega_{3}+\omega_{5}^{\prime}+\omega_{6}^{\prime} & \omega_{7}+\omega_{8} & \omega_{3}+\omega_{5}-\omega_{6}^{\prime} & \omega_{1}-\frac{1}{84} & 0 & \frac{1+\omega_{2}}{2} \\
\hline 0 & \omega_{12} & \omega_{12}^{\prime} & \frac{8}{25} & \omega_{12}^{\prime} & \omega_{12} & 0 & 1 \\
\frac{1}{42} & 2 \omega_{1} & 2 \omega_{1}^{\prime} & \frac{128}{525} & 2 \omega_{1}^{\prime} & 2 \omega_{1} & \frac{1}{42}
\end{array}
$$$$
\left[\omega_{1}=\frac{124-7 \sqrt{15}}{1400}, \quad \omega_{1}^{\prime}=\frac{124+7 \sqrt{15}}{1400}, \quad \omega_{2}=\sqrt{\frac{15+2 \sqrt{15}}{33}}, \quad \omega_{2}^{\prime}=\sqrt{\frac{15-2 \sqrt{15}}{33}},\right.
$$$$
\omega_{3}=\frac{413}{363} \omega_{1}^{\prime}, \quad \omega_{3}{ }^{\prime}=\frac{413}{363} \omega_{1}, \quad \omega_{4}=\frac{54+5 \sqrt{15}}{2178}, \quad \omega_{4}{ }^{\prime}=\frac{54-5 \sqrt{15}}{2178}, \quad \omega_{5}=\frac{98+27 \sqrt{15}}{1320} \omega_{2},
$$$$
\omega_{5}^{\prime}=\frac{98-27 \sqrt{15}}{1320} \omega_{2}^{\prime}, \quad \omega_{6}=\frac{658+199 \sqrt{15}}{14520} \omega_{2}, \quad \omega_{6}^{\prime}=\frac{658-199 \sqrt{15}}{14520} \omega_{2}^{\prime}, \quad \omega_{7}=\frac{3276-100 \sqrt{15}}{27225},
$$$$
\omega_{7}^{\prime}=\frac{3276+100 \sqrt{15}}{27225}, \quad \omega_{8}=\frac{36-4 \sqrt{15}}{165} \omega_{2}, \quad \omega_{8}^{\prime}=\frac{36+4 \sqrt{15}}{165} \omega_{2}^{\prime}, \quad \omega_{9}=\frac{8-\sqrt{15}}{60} \omega_{2},
$$$$
\omega_{9}^{\prime}=\frac{8+\sqrt{15}}{60} \omega_{2}^{\prime}, \quad \omega_{10}=\frac{861-73 \sqrt{15}}{9600}, \quad \omega_{10}^{\prime}=\frac{861+73 \sqrt{15}}{9600}, \quad \omega_{11}=\frac{111-17 \sqrt{15}}{640} \omega_{2},
$$$$
\left.\omega_{11}^{\prime}=\frac{111+17 \sqrt{15}}{640} \omega_{2}^{\prime}, \quad \omega_{12}=\frac{51+2 \sqrt{15}}{300}, \quad \omega_{12}^{\prime}=\frac{51-2 \sqrt{15}}{300}\right] .
$$ 
study of the error terms of I-processes and II-processes it is convenient to classify the elementary differentials of an even order in a similar sort of way.

Consider $\mathbf{F}=\left\{\mathbf{F}_{1} \mathbf{F}_{2} \cdots \mathbf{F}_{s}\right\}$ of order $r=2 \nu$ and suppose that $r_{1}$ is not exceeded by any of $r_{2}, r_{3}, \cdots, r_{s} . \mathbf{F}$ is said to be

$$
\begin{aligned}
& \text { semi-central if } r_{1} \leqq \nu, \\
& \text { quasi-central if } r_{1}=\nu, \\
& \text { bi-central if } r_{1}<\nu .
\end{aligned}
$$

Thus if $\mathbf{F}$ is semi-central it is either quasi-central or bi-central but not both. If $\mathbf{F}$ is not semi-central it is neither quasi-central nor bi-central. In this case suppose $\mathbf{F}_{1}=\left\{\overline{\mathbf{F}}_{1} \overline{\mathbf{F}}_{2} \cdots \overline{\mathbf{F}}_{\sigma}\right\}$ and define

$$
\mathbf{F}^{\prime}=\left\{\overline{\mathbf{F}}_{1} \overline{\mathbf{F}}_{2} \cdots \overline{\mathbf{F}}_{\sigma}\left\{\mathbf{F}_{2} \mathbf{F}_{3} \cdots \mathbf{F}_{s}\right\}\right\} .
$$

In the same way as with $G$-processes we can form $F, F^{\prime}, F^{\prime \prime}, \cdots$ until $F^{*}$ is reached which is semi-central. Note, however, that if $\mathbf{F}$ is quasi-central then so is $\mathbf{F}^{\prime}$ so we do not necessarily reach an $\mathrm{F}^{*}$ which is bi-central.

Corresponding to Theorems 11, 12, 13 of [2] we now state the principal results of this section. For the statements numbered I, II or III it is to be understood that a I-process, a II-process or a III-process is assumed. In each case $\nu$ is the number of stages.

Theorem 4.

I: If $\mathrm{F}=\left\{\mathrm{f}^{2 \nu-1}\right\}, \delta=-(\nu !)^{2}[(\nu-1) !]^{2} /[(2 \nu) !(2 \nu-1) !]$,

II: If $\mathbf{F}=\left\{\mathrm{f}^{2 \nu-1}\right\}, \delta=(\nu !)^{2}[(\nu-1) !]^{2} /[(2 \nu) !(2 \nu-1) !]$,

III: If $\mathbf{F}=\left\{\mathbf{f}^{2 \nu-2}\right\}, \delta=\nu ![(\nu-1) !]^{2}(\nu-2) ! /[(2 \nu-1) !(2 \nu-2) !]$.

THEOREM 5 .

I: If $\mathbf{F}$ is semi-central, $\delta=-(\nu !)^{2}[(\nu-1) !]^{2} /\left\{[(2 \nu-1) !]^{2} \gamma\right\}$,

II: If $\mathbf{F}$ is bi-central, $\delta=(\nu !)^{2}[(\nu-1) !]^{2} /\left\{[(2 \nu-1) !]^{2} \gamma\right\}$,

If $\mathbf{F}$ is quasi-central, $\delta=-(\nu !)^{2}[(\nu-1) !]^{2} /\left\{[(2 \nu-1) !]^{2} \gamma\right\}$,

III: If $\mathbf{F}$ is central, $\delta=\nu ![(\nu-1) !]^{2}(\nu-2) ! /\left\{[(2 \nu-2) !]^{2} \gamma\right\}$.

THEOREM 6.

I, II: If $\mathbf{F}$ is not semi-central and $\mathbf{F}^{\prime}$ is defined by (3) then $\delta=-\delta^{\prime}$,

III: If $\mathbf{F}$ is non-central and $\mathbf{F}^{\prime}$ is defined as in [2] then $\delta=-\delta^{\prime}$.

To prove Theorem 4 we write $p_{0}+p_{1} c+p_{2} c^{2}+\cdots+p_{\nu} c^{\nu}$ for the polynomial whose roots are $c_{1}, c_{2}, \cdots c_{\nu}$. We have the equations $B(2 \nu-d-1)$ where $d=0$ (I or II) or $d=1$ (III). That is, we have the equations

$$
\sum_{i=1}^{\nu} b_{i} c_{i}^{k-1}=1 / k, \quad k=1,2, \cdots, 2 \nu-d-1 .
$$

Also we have the equation

$$
\sum_{i=1}^{\nu} b_{i} c_{i}^{2 \nu-d-1}=\frac{1}{2 \nu-d}+\delta .
$$
find

Multiplying the first $\nu+1$ of these equations by $p_{0}, p_{1}, \cdots, p_{\nu}$ and adding, we

$$
p_{0}+p_{1} / 2+p_{2} / 3+\cdots+p_{\nu} /(\nu+1)=0 \text {. }
$$

Similarly,

$$
p_{0} / 2+p_{1} / 3+p_{2} / 4+\cdots+p_{\nu} /(\nu+2)=0
$$


INTEGRATION PROCESSES BASED ON RADAU QUADRATURE FORMULAS 239

$$
p_{0} / 3+p_{1} / 4+p_{2} / 5+\cdots+p_{\nu} /(\nu+3)=0
$$

$p_{0} /(\nu-d)+p_{1} /(\nu-d+1)+p_{2} /(\nu-d+2)+\cdots+p_{\nu}\left[\frac{1}{2 \nu-d}+\delta\right]=0$.

We have the additional restriction $c_{1}=0$ and hence $p_{0}=0$ for cases I and III. Also we have $c_{\nu}=1$ and hence

$$
p_{0}+p_{1}+p_{2}+\cdots+p_{v}=0
$$

for cases II and III.

Eliminating $p_{0}, p_{1}, p_{2}, \cdots, p_{\nu}$ in the various cases we find

$$
\begin{aligned}
\delta & =-D_{\nu+1}^{(\mathrm{I})} / D_{\nu}{ }^{(\mathrm{I})}, \\
\delta & =-D_{\nu+1}^{(\mathrm{II})} / D_{\nu}{ }^{(\mathrm{II})}, \\
\delta & =-D_{\nu+1}^{(\mathrm{III})} / D_{\nu}{ }^{(\mathrm{III})},
\end{aligned}
$$

III:

where we have written

$$
\begin{aligned}
& D_{N}^{(\mathrm{I})}=\left|\begin{array}{cccc}
\frac{1}{2} & \frac{1}{3} & \cdots & \frac{1}{N} \\
\frac{1}{3} & \frac{1}{4} & \cdots & \frac{1}{N+1} \\
\vdots & \vdots & & \vdots \\
\frac{1}{N} & \frac{1}{N+1} & \cdots & \frac{1}{2 N-2}
\end{array}\right|, \\
& D_{N}^{(\mathrm{II})}=\left|\begin{array}{cccc}
1 & 1 & \cdots & 1 \\
1 & \frac{1}{2} & \cdots & \frac{1}{N} \\
\frac{1}{2} & \frac{1}{3} & \cdots & \frac{1}{N+1} \\
\vdots & \vdots & & \vdots \\
\frac{1}{N-1} & \frac{1}{N} & \cdots & \frac{1}{2 N-2}
\end{array}\right|, \\
& D_{N}^{(\mathrm{III})}=\left|\begin{array}{cccc}
1 & 1 & \cdots & 1 \\
\frac{1}{2} & \frac{1}{3} & \cdots & \frac{1}{N} \\
\frac{1}{3} & \frac{1}{4} & \cdots & \frac{1}{N+1} \\
\vdots & \vdots & & \vdots \\
\frac{1}{N-1} & \frac{1}{N} & \cdots & \frac{1}{2 N-3}
\end{array}\right| .
\end{aligned}
$$


We also write as in [2]

$$
D_{N}=\left|\begin{array}{cccc}
1 & \frac{1}{2} & \ldots & \frac{1}{N} \\
\frac{1}{2} & \frac{1}{3} & \cdots & \frac{1}{N+1} \\
\vdots & \vdots & & \vdots \\
\frac{1}{N} & \frac{1}{N+1} & \cdots & \frac{1}{2 N-1}
\end{array}\right| .
$$

In $D_{N}$ we subtract the last from each of the other rows and rearrange to find

$$
D_{N}=(-1)^{N+1} \frac{[(N-1) !]^{2}}{(2 N-1) !} D_{N}{ }^{(\mathrm{II})}
$$

In $D_{N}{ }^{\text {(II) }}$ we subtract the last from each of the other columns and rearrange to find

$$
D_{N}{ }^{(\mathrm{II})}=(-1)^{N+1} \frac{[(N-1) !]^{2}}{(2 N-2) !} D_{N-1} \text {. }
$$

From these we deduce that for the II-process

$$
\delta=(\nu !)^{2}[(\nu-1) !]^{2} /[(2 \nu) !(2 \nu-1) !]
$$

and for the G-process

$$
\delta=-D_{\nu+1} / D_{\nu}=-(\nu !)^{4} /[(2 \nu) !(2 \nu+1) !] .
$$

In $D_{N}$ we subtract the first row from each of the other rows and rearrange to find

$$
D_{N}=\frac{(-1)^{N+1}}{N} D_{N+1}^{(\mathrm{III})}
$$

which enables us to deduce that for a III-process

$$
\delta=\nu ![(\nu-1) !]^{2}(\nu-2) ! /[(2 \nu-1) !(2 \nu-2) !]
$$

Finally in $D_{N}{ }^{(\mathrm{III})}$ we subtract the last column from each of the other columns and rearrange to find

$$
D_{N}{ }^{(\mathrm{III})}=(-1)^{N} \frac{(N-1) !(N-2) !}{(2 N-3) !} D_{N-1}^{(\mathrm{I})}
$$

which enables us to deduce that for the I-process

$$
\delta=-(\nu !)^{2}[(\nu-1) !]^{2} /[(2 \nu) !(2 \nu-1) !] .
$$

The proof of Theorem 5 is analogous to the proof of Theorem 12 of [2]. It is easy to show that $\delta \gamma$ is constant among the semi-control $\mathrm{F}$ of order $2 \nu$ (I-processes); that $\delta \gamma$ is constant ( $=C_{1}$ say) among the bi-central $\mathbf{F}$ of order $2 \nu$ and constant ( $=C_{2}$ say) among the quasi-central $\mathbf{F}$ of order $2 \nu$ (II-processes); and that $\delta \gamma$ is constant among the central $\mathbf{F}$ of order $2 \nu-1$ (III-processes). It remains to show that for II-processes $C_{1}+C_{2}=0$. 
To prove this we consider the quasi-central example

$$
\mathbf{F}=\left\{\mathbf{f}^{\nu-1}\left\{\mathbf{f}^{\nu-1}\right\}\right\}
$$

for which $\gamma=2 \nu^{2}$. We have

$$
\begin{aligned}
{\left[\phi^{\nu-1}\left[\phi^{\nu-1}\right]\right] } & =\sum_{i, j=1}^{\nu} b_{i} c_{i}^{\nu-1} a_{i j} c_{j}^{\nu-1} \\
& =\sum_{j=1}^{\nu} b_{j}\left(1-c_{j}{ }^{\nu}\right) c_{j}^{\nu-1} \\
& =\frac{1}{2 \nu^{2}}-\frac{\delta_{0}}{\nu}
\end{aligned}
$$

where $\delta_{0}$ corresponds to $\Phi_{0}=\left[\phi^{2 v-1}\right]$. Thus

$$
\delta=-\frac{\delta_{0}}{\nu}
$$

and hence, since $\gamma_{0}=2 \nu$ we have

$$
\gamma \delta=-\gamma_{0} \delta_{0} .
$$

The proof of Theorem 6 is analogous to the proof of Theorem 13 of [2] and need not be given here.

These theorems enable us to write down the values of $\delta$ and $\epsilon=\beta \delta /(r-1)$ ! for the various $\mathbf{F}$ of order $r=2 \nu$ (I or II) or $r=2 \nu-1$ (III). Moreover, there are two consequences of these results which will be found useful if error estimates are to be made during a calculation.

CoROLlaRY 1. The error vectors for I-processes and II-processes can be written in the forms

$$
\begin{aligned}
& (\mathbf{A}+\mathbf{B}) h^{2 \nu}+O\left(h^{2 \nu+1}\right) \\
& (\mathbf{A}-\mathbf{B}) h^{2 \nu}+O\left(h^{2 \nu+1}\right)
\end{aligned}
$$

where $\mathbf{A}, \mathbf{B}$ each consist of terms of the form $\epsilon \mathbf{F}$ and no $\mathbf{F}$ occurs in both $\mathbf{A}$ and $\mathbf{B}$.

Corollary 2. If the error vector for the $\nu-1$ stage $G$-process is

$$
\mathrm{C} h^{2 \nu-1}+O\left(h^{2 \nu}\right)
$$

then the error vector for the $\nu$ stage III-process is

$$
-\frac{\nu}{\nu-1} \mathbf{C} h^{2 \nu-1}+O\left(h^{2 \nu}\right) \text {. }
$$

4. Practical Computing Procedures. There are two situations in which the methods suggested here and in [2] would be worth serious consideration as the basis of practical computing schemes. The first is where a self-starting method is required and where the accuracy demanded makes a process of high order desirable. Secondly we might have equations for which the cost of computing $f(y)$ for a given $x$ value (one of the components of the $y$ vector may be taken as $x$ ) is high compared with the cost of repeating this calculation with $\mathrm{y}$ changed but $x$ unchanged. 
In this second situation the implicit nature of the processes is relatively less of an objection to their use. If the equations are linear the iterations may, in fact, be bypassed by the use of standard linear-algebraic techniques.

However, even when other considerations point to the use of some Runge-Kutta process the difficulty of estimating the truncation error is a serious objection to this choice. With processes of high order, error estimates based on the methods of Bieberbach [6] or Lotkin [7] are difficult to apply and are likely to overestimate the error considerably [8].

We now suggest two computing procedures in which an estimate of the truncation error can be made during the calculation. Let $\mathrm{y}_{1}, \mathrm{y}_{2}, \cdots$ be the true result vectors corresponding to the point $x_{0}+h, x_{0}+2 h, \cdots$. The first method is to apply a I-process to $\mathrm{y}_{0}$ to find $\mathrm{y}_{1}$ and then a II-process (with the same number of stages) to $\mathrm{y}_{1}$ to find $\mathrm{y}_{2}$. In the first step the error committed is

$$
(\mathbf{A}+\mathbf{B}) h^{2 \nu}+O\left(h^{2 \nu+1}\right)
$$

and in the second

$$
(\mathbf{A}-\mathbf{B}) h^{2 \nu}+O\left(h^{2 \nu+1}\right)
$$

so that the error in the estimate of $\mathrm{y}_{2}$ will be

$$
2 \mathbf{A} h^{2 v}+O\left(h^{2 v+1}\right) \text {. }
$$

This error can be estimated by applying a II-process to $\mathrm{y}_{1}$ but with the sign of $h$ changed. This will estimate $\mathrm{y}_{0}$ but with the error (4). Thus we can find $\mathrm{y}_{2}$ to terms in $h^{2 v}$ by subtracting this error estimate from the previously found value of $\mathrm{y}_{2}$. Moreover, assuming the truncation error changes slowly we can use this same error estimate with $\mathbf{y}_{2}, \mathbf{y}_{4}, \cdots$ recalculating it after a certain number of steps.

Note that for the II-process with $h$ reversed $\mathbf{g}^{(1)}, \mathbf{g}^{(2)}, \cdots, \mathbf{g}^{(\nu)}$ correspond approximately to $\mathbf{g}^{(\nu)}, \mathbf{g}^{(\nu-1)}, \cdots, \mathbf{g}^{(1)}$ already found in the forward moving I-process and these values may be used to start the iteration for the II-process.

The second computing procedure uses the III-process of $\nu$ stages for the basic method. Here the check on the truncation error can be made by applying the G-process of $\nu-1$ stages over an interval already treated by the III-process. If the error in the III-process is

$$
-\frac{\nu}{\nu-1} \mathbf{C} h^{2 \nu-1}+O\left(h^{2 \nu}\right)
$$

then the error for the G-process is

$$
\mathrm{Ch}^{2 \nu-1}+O\left(h^{2 \nu}\right) \text {. }
$$

Thus if the two computations give $y^{(\mathrm{III})}$ and $\mathrm{y}^{(\mathrm{G})}$ then the error in $\mathrm{y}^{(\mathrm{III})}$ is approximately given by

$$
\frac{\nu}{2 \nu-1}\left(\mathrm{y}^{(\mathrm{III})}-\mathrm{y}^{(\mathrm{G})}\right)
$$

4. Numerical Examples. To illustrate the use of the I-process and the II-process with $\nu=2$ we consider the differential equation 


$$
y^{\prime}=x y, \quad y(0.5)=1
$$

with solution $y=\exp \frac{1}{2}\left(x^{2}-\frac{1}{4}\right)$. Using $h=.1$ for a I-process starting at the initial point we find $g_{1}=.5$ and, after 5 iterations starting from $g_{2}=0, g_{2}=.5872027$. Thus $y(.6)=1.05654020$ is found and this differs from $\exp (0.55)=1.05654061$ by $-41 \cdot 10^{-8}$.

With the same value of $h$, a II-process starting from $x=.6$ gives $g_{1}=.6835731$, $g_{2}=.7874283$ so that $y(.7)=1.12749389(\exp (.12)=1.2749685)$, in error by $-296.10^{-8}$.

To estimate the error in $y(.7)$ we start from $x=.6$ with $h=-.1$ and use a II-process to estimate $y(.5) ; g_{1}$ is found to be .5876069 ( 3 iterations starting from $g_{1}=.5872027$ as found during the forward step from .5 as $\left.g_{2}\right)$ and $g_{2}=.4988898$ so that $y(.5)=1-256.10^{-8}$. Subtracting $-256.10^{-8}$ from the previously found value of $y(.7)$ we obtain 1.12749645 so that most of the error is now removed.

Although in most cases the principal error function is too complicated to make possible an a priori error estimate, with $\nu=2$ it is for the I-process

$$
\frac{h^{4}}{72}\left(\left\{{ }_{3} \mathbf{f}\right\}_{3}-\{\{\mathbf{f}\} \mathbf{f}\}+\left\{{ }_{2} \mathbf{f}\right\}_{2}-\frac{1}{3}\left\{\mathbf{f}^{3}\right\}\right)
$$

and for the II-process

$$
\frac{h^{4}}{72}\left(\left\{{ }_{3} \mathbf{f}\right\}_{3}-\{\{\mathbf{f}\} \mathbf{f}\}-\left\{{ }_{2} \mathbf{f}\right\}_{2}+\frac{1}{3}\left\{\mathbf{f}^{3}\right\}\right) .
$$

With the equation $y^{\prime}=x y$, evaluation of the $y$ component of these functions gives

$$
\begin{aligned}
& \frac{h^{4}}{72} y\left(x^{4}+2 x^{2}-1\right), \\
& \frac{h^{4}}{72} y\left(x^{4}-2 x^{2}-1\right) .
\end{aligned}
$$

II:

Between $x=.5$ and $x=.7$ these functions do vary somewhat but they still furnish reasonable error estimates if evaluated at $x=.5, y=1$. For the I-process the error estimate is $\left(10^{-4} / 72\right) \cdot(-7 / 16)=-61.10^{-8}$ and for the II-process it is $\left(10^{-4} / 72\right)$. $(-23 / 16)=-200.10^{-8}$. Correcting the original estimates of $y(.6)$ and $y(.7)$ we obtain $y(.6)=1.05654081, y(.7)=1.12749650$ so that again most of the error is removed.

An example of the use of the G-process with $\nu=3$ was given in [2]. In this example the equation $y^{\prime}=y, y(0)=1$ was integrated from $x=0$ to $x=.3$ in a single step. We now give results for the same step performed using the III-process with $\nu=4 . g_{1}=1$ was found explicitly and after 8 iterations starting with $g_{2}=$ $g_{3}=1$ it was found that $g_{2}=1.0864494560, g_{3}=1.2424541986$. An explicit evaluation gives $g_{4}=1.349833887$ so that $y(.3)=1.3498588040=y^{(\mathrm{III})}$ using a notation from Section 3. The value found in [2] was $y^{(\mathrm{G})}=1.3498588105=y^{(\mathrm{III})}+65.10^{-10}$. Hence, the error in $y^{(\mathrm{III})}$ is approximately $-\frac{4}{7} \cdot 65 \cdot 10^{-10}=-37.10^{-10}$. Correcting $y^{(\text {III) }}$ we find $y(.3)=1.3498588077$ in close agreement with $\exp (.3)=1.3498588076$. 
1. J. C. Butcher, "Coefficients for the study of Runge-Kutta integration processes," J. Austral. Math. Soc., v.3, 1963, p. 185-201.

2. J. C. Butcher, "Implicit Runge-Kutta processes," Math. Comp., v.18, 1964, p. 50-64.

3. M. RADAU, "Étude sur les formules d'approximation qui servent à calculer la valeur numérique d'une intégrale définie," J. Math. Pures Appl., s.3, v.6, 1880, p. 283-336.

4. P. Henricr, Discrete variable methods in ordinary differential equations, ist edition, Wiley, New York, 1962.

5. P. C. Hammer \& J. W. Hollingsworth, "Trapezoidal methods of approximating solutions of differential equations," $M T A C$, v.9, 1959, p. 92-96. 1944.

6. L. Bieberbach, Theorie der Differentialgleichungen, Dover Publications, New York,

7. M. Lotkin, "On the accuracy of Runge-Kutta's! method," MTAC, v.5, 1951, p. 128-132.

8. H. A. Antosiewicz \& W. GaUtschi, "Numerical methods in ordinary differential equations," in A Survey of Numerical Analysis (Editor, J. TodD), McGraw-Hill Book Company, New York, 1962. 